\title{
Non-functional biomimicry: Utilising natural patterns in order to provoke attention responses
}

\begin{abstract}
Natural reoccurring patterns arise from chaos and are prevalent throughout nature. The formation of these patterns is controlled by, or produces, underlying geometrical structures. Biomimicry is the study of nature's structure, processes and systems, as models and solutions for design challenges and is being widely utilized in order to address many issues of contemporary engineering. Many academics now believe that aesthetics stem from pattern recognition, consequently, aesthetic preference may be a result of individuals recognising, and interacting with, natural patterns. The goal of this research was to investigate the impact of specific naturally occurring pattern types (spiral, branching, and fractal patterns) on user behaviour; investigating the potential of such patterns to control and influence how individuals interact with their surrounding environment. The results showed that the underlying geometry of natural patterns has the potential to induce attention responses to a statistically significant level.
\end{abstract}

\section{Keywords}

Biomimicry, product aesthetics, non-functional biomimicry, natural patterns 


\section{Introduction}

Aesthetics is the philosophical study of beauty, art, and the nature of the appreciation of beauty. Many academics believe that aesthetics stem from pattern recognition based on the brain's evolutionary adaptations. Rhodes et al. (1998) argue that the human brain associates certain patterns with beauty, maintaining that facial symmetry is fundamental to the perception of beauty. They argue that there is a correlation between symmetry and fitness in nature, especially in reproductive partners, and, as a result, symmetry is selected due to the increased survival advantage it offers the preceding generation. Enquist \& Arak (1994) agree that the human brain is programmed to be attracted to symmetry, however, they argue that the basis for the attraction is due to the advantage it offers humans with regard to object recognition. They claim that such preferences allow the brain to recognise objects regardless of their orientation within a visual field.

There are recurring patterns that manifest throughout nature, one example of this is the Fibonacci sequence. The Fibonacci sequence is a sequence where $\mathrm{N}$ is the sum of the two preceding numbers, i.e. $0,1,1,2,3,5,8 \ldots$. It is not only abundant in nature, but it has already formed the basis for many aesthetic designs (Fig 1). Teuscher (2004) explains mathematician Alan Turing believed that such patterns are a result of living matter's ability to self-organise. Turing used nonlinear differential equations to create a computer model of nature's hypothesised ability to self-organise. In 1979 mathematician Benoit Mandelbrot created the Mandelbrot set (Fig 2), a fractal set of points which demonstrates the creation of complex selfsimilar structures from simple mathematical rules.

The aim of this research was to investigate the impact of specific, naturally occurring pattern types (spiral, branching, and fractal patterns) on user behaviour; investigating the potential of such patterns to control and influence how individuals interact with their surrounding environment through evoking attention responses in individuals. An experiment was carried out using the virtual world 'Second Life' with the purpose of examining how individuals would react to different patterns within an environment. The results showed that the underlying geometry of natural patterns has the potential to evoke attention responses to a statistically significant level.

\section{Patterns in nature}

Philip Ball (Ball \& Borley, 1999; Ball 2008, Ball, 2011) argues that complexity is controlled by, and is the result of, simple physical laws. The theory that simple mathematical equations could explain growth patterns in nature was pioneered by Thompson (1915) in his influential book 'On growth and form'. Ball explains the mathematical concepts behind pattern formation and details the emergent properties of certain patterns that lead to complexity from simplicity.

Hanzen (2009) believes that three principles direct pattern configuration in living and nonliving systems:

A) Patterns emerging as a result of interactions involving numerous entities, e.g. molecules, sand, etc. 
B) Groupings formed through the combination of such entities.

C) Selection of functional configurations of entities.

Hanzen et al. (2007) demonstrated that the methodological evolutionary principles of pattern configuration in biological life forms can be traced all the way down to RNA configuration. Camazine et al. (2001) share Hanzen's belief that evolution is the guiding force for pattern selection in living systems and they also echo his opinions on pattern formation through selforganisation. They recognise, however, that there may be cases where patterns in nature emerge which are imposed by alternative sources, for example, following a leader. Nonetheless, Camazine et al. argue that such patterns are emergent properties of complex systems which are reliant on self-organisation and, therefore, all patterns have a base in self-organisation.

Turing patterns are patterns in nature which are formed through Hanzen's second principle. Several studies have been carried out into Turing patterns (Figure 1), such as that of Millonas \& Rauch (2004) and Ouyang \& Swinney (1991) which demonstrate the ability of such patterns to self-organise, the mechanism being the diffusion of certain molecules over cell membranes. They go on to explain that the patterns are defined by feedback loops caused by self-replicating chemicals. There is strong evidence to suggest that such pattern formation is the cause of markings in the skin of certain mammals, e.g. leopards, cows. For example, research carried out by Lui et al. (2006) recreated the exact growth of the markings on a Jaguar's coat, throughout its development to adulthood, using Turing Patterns. They also presented strong evidence which suggests Turing patterns may be responsible for patterns in bacteria, fish, insects, and many other organisms. Rietkerk \& Koppel (2008) collated and reviewed several studies which hypothesise that feedback loops, such as those seen in Turing patterns, may, in fact, be influencing entire ecosystems with the organisms being the self-replicating agents. However, they concede that further studies are required in order to gain a fuller understanding of pattern formations at such a level.

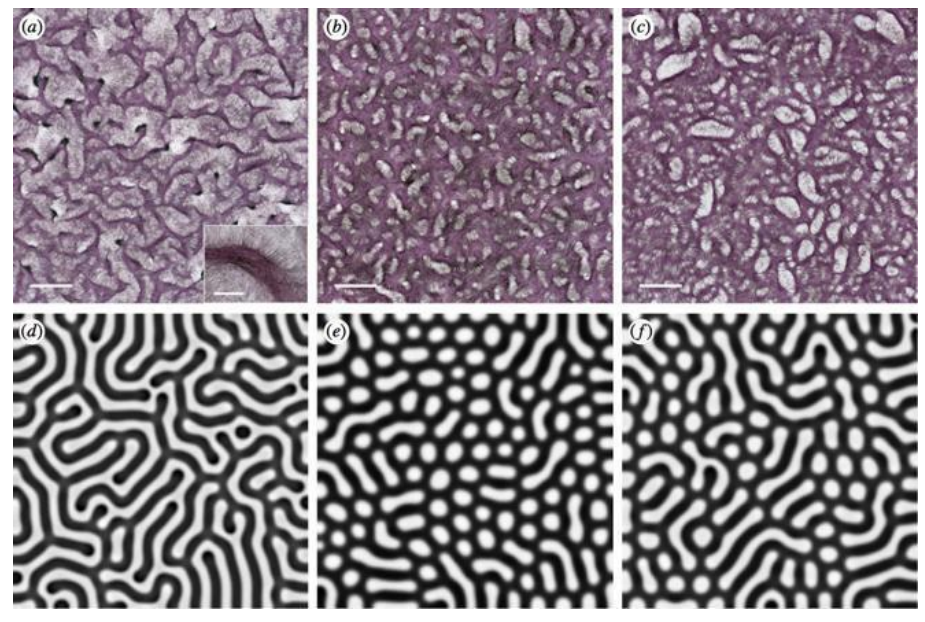

Figure 1: Turing patterns for stable concentrations of activator and inhibitor in a two-dimensional array of cells. Branching Patterns (Figure 2) are also widely observed throughout nature. Pickett \& White (2011) detail the evidence which suggests that branching patterns are a result of mathematical 
functions that minimise the total length of all the stems in the system. They suggest such patterns are retained in trees in order to allow them to collect the maximum amount of sunlight with the most effective possible structure. Their hypothesis is supported by other structures found in nature, for example, the human lung, where the branching pattern maximises the surface areas of the blood stream for diffusion. Such patterns can also be seen in multiorganism systems, for example, ants. Holldolber \& Wilson (1990) elucidate that driver ants follow branching patterned chemical pathways while hunting; this allows them to cover them to cover the maximum possible area in the most in the most effective manner. The origins of the patterns, however, are still a cause for debate. Dawkins (1986) argues that such patterns are the result of natural selection, however, clearly rivers and lighting are not subject to natural selection. Leopold (date?) concedes to the objection that branching patterns are not completely universal throughout nature and that under certain conditions alternative structures are selected, for example, desert trees.
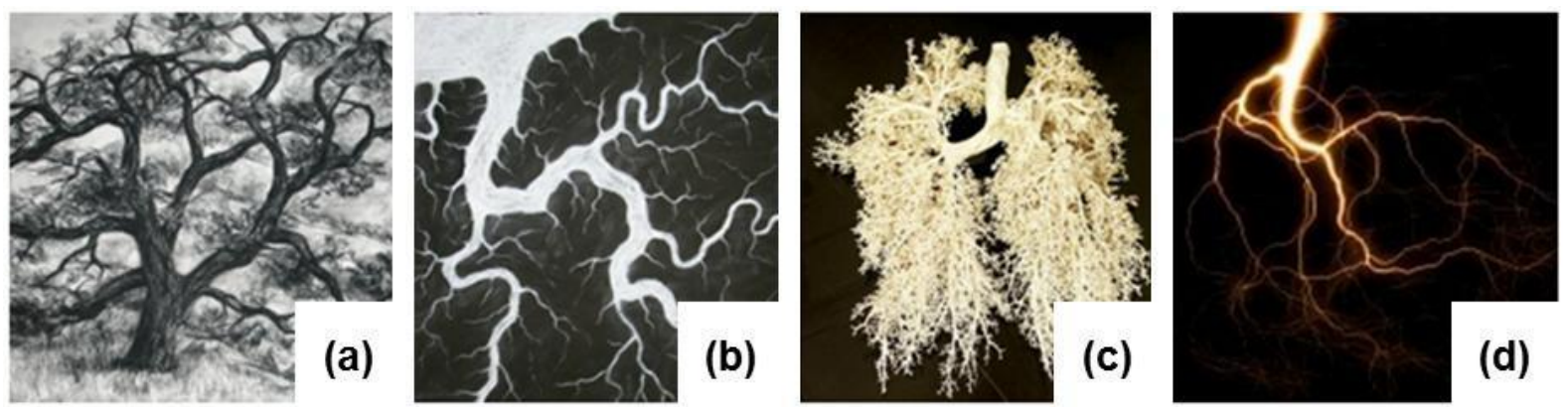

Figure 2: Branching patterns observed in trees (a), rivers (b), the human lung (c) and lightening (d).

Spiral patterns are also abundant throughout nature, appearing in, for example, galaxy formation, plant structures, storms, and shells. Cook (1979), whose work is accepted by contemporary scientists (Steadman, 2008; Livio, 2008), suggests that spirals are found throughout the universe due to equilibrium and their ability to conserve energy. Cook, however, suggests that biological organisms make use of spirals for a variety of reasons, for example, fitting organs into small areas while maximising the surface area of the organ, like the long intestine of frogs which resembles a tightly coiled spring in tadpoles.

Many scientists now argue that such patterns are the result of fractals. Fractals are sets which contain a fractal dimension. The fractal dimension changes with scale resulting in self similar patterns which are greater in size than the space they are contained in. Mandelbrot (1983), a key author in the field who later presented the case for nature being controlled by fractal geometry, created a fractal set which demonstrates the ability to create complex self-similarity from simple mathematical rules. The significance of this demonstration is emphasised by authors such as Peitgen \& Richter (1986) and Devaney (1999) who explain that one of nature's key sequences is found within the Mandelbrot set: the Fibonacci sequence. Figure 3 (a) illustrates how the shape of the Mandelbrot set varies as the algorithms are repeated and as the set expands, or 'grows', the shapes follow a distinctive pattern in concordance to the Fibonacci sequence.

Meinhardt (1995) explains that many researchers now believe that the Fibonacci sequence controls many aspects of the growth of organisms. Dunlap (1999) believes this is particularly true in the case of spiral patterns, as Fibonacci spirals can be found in many aspects of nature, 
ranging from plants to animal shells'. Dunlap further hypothesizes that spiral patterns may also, ultimately, be responsible for the branching patterns of trees, as shown in Figure 3(b). However, this is based on the ability to superimpose spirals over tree patterns and a sceptic would rightly claim that this could be the result of apophenia which, as explained by Paul et al. (2011), is the tendency for humans to see patterns in information where no such patterns exist. Undoubtedly, while numerous patterns could be imposed over trees, correlation does not imply causation. Nevertheless, the Fibonacci patterns and spirals can be observed throughout nature and the cosmos.

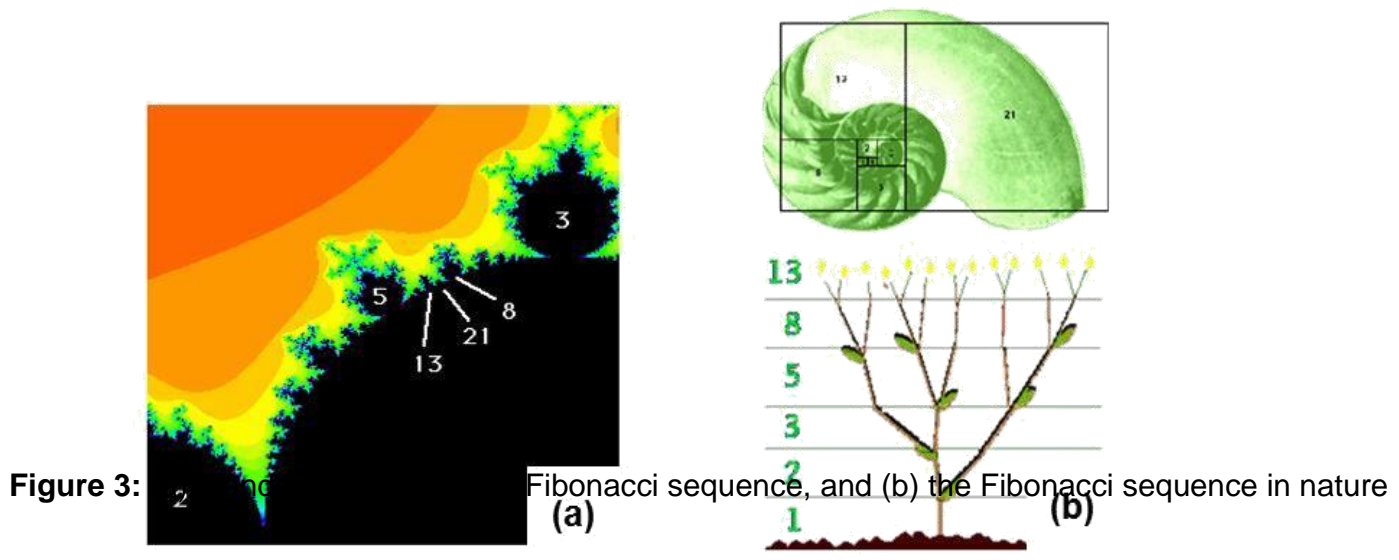

\section{Natural aesthetics}

Research, such as that carried out by Chamberlain (2000) and Bottino \& Laurentini (2010), allows the evolution of aesthetics to be understood in regard to the main evolutionary factors. Dawkins (2006), explains that the definitive success of a gene is in its ability to reproduce. Ultimately, a gene that can out-reproduce its rivals will propagate throughout a population regardless of any potential side effects.

As outlined by Williams (1966), as far as modern mammals are concerned, including Homo sapiens, success is dependent on the following main evolutionary factors:

- Prey acquisition (finding food/water)

- Predator avoidance (avoiding becoming food)

- Necessities (shelter, remaining healthy, etc.)

- Reproduction (successfully propagating one's genes)

Any psychological drive that can improve any aspect of the above will lead to a genetic advantage.

Chamberlain (2000) investigated humans' psychological preference for savannah environments, suggesting that savannah environments would have benefited early hominids in respect to two of the highlighted factors: prey acquisition and predator avoidance. The open nature of the environment would have been easy to navigate and allowed them to spot and catch prey, while trees would have allowed them refuge from large predators like lions. Joye 
et al. (2011) suggest that such environments have a substantial physiological impact on humans in reducing stress and increasing happiness. They elucidate that savannah environment emulation is now a vital part of landscape design and is currently a major feature of retail and business environment design. There still, however, remains much disagreement over the preference for savannah settings and although Falk \& Bailing's 2010 experiment was a reproduction of an earlier experiment (Baling \& Falk, 1982), other researchers, such as Han (2007), have tried and failed to reproduce their results.

Nevertheless, people do show a preference for natural environments and prominent authors Orians \& Heerwagen emphasise a particular affinity to vegetation (Orians, 1986; Orians \& Heerwagen, 1992; Heerwagen \& Orians 1993). They detail various aspects of trees that could benefit human survival and, therefore, the ability to reproduce, for example, trees can provide food, safety and shelter. They hypothesise that individuals who evolved a psychological attraction to trees would spend more time in their vicinity; this would have led to an increasing change of being able to exploit their resources which, in turn, would have provided an evolutionary advantage.

Joye et al. (2011) take issue with the above findings of Orians \& Heerwagen, as their findings show that humans have a psychological preference for any type of 'greenery'. They suggest that if psychological drives were a result of evolution, then one would expect to see a more specific psychological attraction, for example, to specific resource wielding trees. They concede, however, that Orians \& Heerwagen have attempted to address this complaint when they presented attraction to flowers as a mechanism for indicating the availability of certain resources, for example, fruit.

Ulrish et al. (1991) carried out an excellent investigation into the impact of natural settings on stress and emotional discomfort. The study involved monitoring the emotional states of 120 individuals, such as, pulse rates, skin conductance, blood pressure etc. The natural environments all contain water and, as their previous studies indicated, Western groups associate water with relaxing natural environments. The individuals were subjected to distressing video footage followed by footage of natural or urban environments. The results showed that the natural environment created a significant decrease in the recovery time of the test subjects in comparison to that of the urban videos. Furthermore, the study uncovered a theme of particular interest: the research showed that, under certain circumstances, individuals exhibited involuntary levels of automatic attention, with the natural environment prolonging periods of involuntary attention.

The evidence demonstrates that humans have deep psychological connections to the resourcerich environment in which the species evolved, including aesthetic attraction. This connection forms the basis of the biophilia hypothesis which suggest that humans have an instinctual connection to other life (Wilson, 1984). Kellert \& Wilson (1995) hypothesise that this connection is driven by biological evolution as, ultimately, it was the manner in which humans interacted with other life forms that guided our evolution. They suggest that, in the same manner that humans have phobias of things which may be detrimental to survival, e.g. snakes and spiders, they also have philias toward that which benefit survival. 
Aesthetic attraction to greenery is of particular interest as tree and plant structures are defined by fractal geometry. Joye argues, supported by previous research (Joye, 2007), that psychological attraction to greenery in landscapes may, in fact, be due to pattern recognition where humans are recognising the underlying fractal geometry. This explanation would also explain aesthetic attraction to greenery as a whole. If individuals are attracted to underlying patterns then it would be reasonable to hypothesise that, under the right conditions, those patterns could be exploited to trigger positive aesthetic reactions.

\subsection{Addressing objections to the science of art}

Although there is wide acceptance within the scientific community that people react positively to certain aesthetic stimulus due to their evolutionary past, there still remains much scepticism toward the ability to directly link evolutionary influences to art in general.

Consequently, Ramachandran \& Hirstein (1999) present a theory of art which suggest that modern art has its foundations in our evolutionary past. They suggest that the logic of art can be broken down into rules and principles which are appreciated due to evolutionary psychology. Although the presented theory is grounded in speculation which is acknowledged by the authors, they argue that the purpose of the paper is to provoke psychologists and evolutionary biologists into further embracing a subject which they feel has not received enough academic scrutiny and they take issue with the fact the many leading academics in certain fields, such as the arts, dismiss the topic as unscientific.

Dutton (2009) presents a detailed case for the evolutionary foundation of art. He suggests it is time that scientists started to view art in relation to natural selection. Chapter five 'Art and Natural Selection' of his book 'The Art Instinct' presents a compelling case for the biological foundations of art. Ultimately, he points to the fact that, in order to understand the biological foundations of art, one must have a clear understanding of Darwinian evolution. Dutton is clearly aware of the attitude of certain members to the academic community towards the biological foundations of art and his opening statement, which is almost certainly a veiled analogy, regarding the refusal of creationists to accept Darwinian evolution is not lost on the discerning reader. Nevertheless, as pointed out by Richards (2001) and in agreement with Ramachandran \& Hirstein (2009) and Dutton there is still resistance to the case that art is grounded in evolution even by defenders of Darwinism (Dutton, 2009).

The objections are invariably based on the ground that art has no 'functional' survival benefit. Dutton rightly points out that it is easy to connect a functional benefit to survival and, therefore, the reproductive success of an organism. For example, it does not take much imagination, nor investigation, to connect the ability to blend into one's environment with a reduced chance of being spotted by predators. Furthermore, one must admit that the link between liking a Picasso painting and reproducing one is certainly not as clear. As pointed out in 4.4, however, it is generally accepted by many leading academics that, in order to fully understand the benefits of functional biomimicry, individuals must look beyond direct analogies and attempt to understand the fundamental principles at work. On these grounds, and as discussed in 4.4, it would seem fair to conclude that one should possess a firm understanding of the underlying 
principles which guide aesthetic preferences prior to dismissing the field. This opinion is supported by Judelman (2004) who complains that there is a significant gap in commutations between art, science, technology, and design.

Authors such as Wannarumon \& Bohez (2006) have suggested a captivating approach to nonfunctional 'artistic' design. They generate jewellery designs using genetic algorithms which evolve through mutation and recombination. The designs are then selected in relation to two 'fitness' criteria. Firstly, they are selected based on their compactness and connectivity which was highlighted above as a method for Fibonacci spirals being utilized by nature to improve organ design. Secondly, they assess the selected designs against aesthetic variables, such as the Golden Ratio.

\subsection{Symmetry and attractiveness}

Perhaps the most prominent, aesthetically pleasing relationship is that of symmetry. Symmetry can be seen throughout design disciplines and is a common aspect of designs ranging from vehicles to websites (Leder \& Carbon, 2005; Sutcliffe, 2001).

A preference of symmetrical features is now widely accepted as having an evolutionary basis with symmetrical features having an influence over the selection of reproductive partners (Jones, 2001; Rhodes et al. 1998; Rhodes et al. 2001; Rhodes, 2006). Prominent author, Rhodes, has carried out several investigations into the area which demonstrate that there is an underlying correlation between facial symmetry and genetic health. One such study investigated the effects of facial symmetry on facial preferences. The research focused on distinctive marks and asymmetry. The results revealed that facial preferences in 17 year olds were directly associated with symmetry, with the majority of asymmetrical faces being perceived as belonging to individuals with poor health and in the case of the male faces the results showed a direct correlation between asymmetrical faces and poor health. Furthermore, Rhodes et al. (1998) revealed there is a direct correlation between facial symmetry and associated beauty. Such results should not be surprising considering nearly all complex life is bilaterally symmetrical and, therefore, symmetry may be considered an indicator of good genes. A study carried out by Rhodes et al. (2005), however, revealed that symmetry was just one of several criteria that influenced sexual preference, with the only significant finding being that women with symmetrical features became sexually active at an earlier age than their peers.

In an evolutionary sense, sexual attractiveness and reproductive success is not a difficult correlation to understand. The association between symmetry and bilaterally symmetrical organisms seems reasonable, however, Chamberlain (2000) rightly complains that mammals are only bilaterally symmetrical in outward appearance only as many of their internal organs are not symmetrical. Enquist and Arak (1994) argue that symmetrical preferences may, in fact, be a by-product of human pattern recognition. They suggest that humans utilize symmetry in order to recognise objects regardless of their orientation and location in one visual field. Chamberlain conceded, however, there is evidence to suggest the genetic symmetry of an animal may serve as a marker for both inner and outer health as, although the inner organs of a mammal are not bilaterally symmetrical, they are the product of the same genes. To this end, facial symmetry may, in fact, be serving as a health marker for the entire genetic makeup of an 
organism. This hypothesis is echoed by Jones et al. (2001) who carried out two studies which produced supporting evidence for the good genes theory.

Conversely, research carried out by Swaddle \& Cuthill (1995) produced results which directly contradict the proposed link between symmetry and attractiveness. They carried out a series of experiments which produced results that show attractiveness decreasing as symmetry increased. This could, however, be ascribed to the manner in which they constructed their experiments. The experiments utilized photos and mirrors to create symmetry. This may have resulted in images which were unrealistic unless the mirrors were placed perfectly and the photos were taken in the correct manner. Zaidel \& Hessamian (2010) elaborate that such studies carried out using modern, digital equipment and photo editing suites which can be used to blend and merge images, invariably showing a correlation between symmetry and attractiveness.

Bottino \& Laurentini (2010) believe that symmetry is only one aspect of facial attractiveness. They maintain that the ideal proportions for a face conform to the golden ratio. They further detail that several of the leading Renaissance artists, such as Leonardo da Vinci and Leon Alberti, documented facial formulas which conform to the ratio. Furthermore, many modern plastic surgeons abide by and support facial standards based on the golden ratio, however, they concede that such opinions have not been backed by empirical studies on facial attractiveness. Nevertheless, it does prompt an interesting question: are we selecting facial preferences due to deeper underlying pattern recognition?

\subsection{Functional Biomimicry}

Prusinkiewicz and Lindenmayer (1991) demonstrated, through computer simulations, the ability of natural patterns, discussed in the previous section, to control the growth and form of complex biological structures. Their research effort was substantial and resulted in impressive computer-generated graphics which closely resembled the growth and appearance of living organisms. Neo-Darwinian evolution, a field which is now supported by overwhelming evidence, explains nature's ability to act on, and determine the evolution of, biological structures. Dawkins (1997), a prominent author in the area of evolutionary biology, presents a comprehensive account of how such biological structures adapt and are selected through Darwinian evolution to solve an immense variety of biological problems. Biological problems which often overlap or resemble issues faced by engineers. As such, entire fields of engineering are arising which are focused on biologically inspired solutions, e.g. bioengineering and bionics. Biomimicry is a term used to encompass biologically inspired design, referring to the examination of nature's models, processes, and principles for inspiration to aid human problems.

Benyus (2002) maintains that biomimicry cannot just improve functional design but can also enhance suitable and eco-friendly design. She argues that, after billions of years of evolution, the natural world has selected that which can last. Furthermore, she believes that emulating aspects of ecosystems is a vital aspect of the future of sustainable engineering. Benyus feels 
strongly regarding the benefits of biomimicry; she argues that biomimicry should be considered its own discipline with its own university departments.

Many authors have echoed Benyus's opinions, such as Passino's (2004) Biomimicry for Optimization, Control, and Automation and Hernandez \& Brebbia (2012) Design \& Nature V: Comparing Design in Nature with Science and Engineering. Klein (2009) believes that Benyus has laid the foundations for biomimicry to emerge as its own discipline. Kaplinsky (2006), however, takes issue with Benyus's book and biomimicry in general.

Tinsley et al. (2007) accept the benefits that biomimicry can bring to engineering, however, they argue that the real benefits or biomimicry will not be realised until engineers view biomimicry as a design methodology as opposed to a tool. They present four case studies which demonstrate a methodology aimed at facilitating the incorporation of biological solutions into non-analogous engineering problems, for example, abscission. Abscission is the processed used by plants to shed dead or damaged leaves. The plant creates a hormone which dissolves the portion of the stem where the leaf is attached.

This process is emulated, in principle, by the Technical University of Denmark who exploit it to assemble micro-screws with a width of $0.6 \mathrm{~mm}$ to plastics. The screws are attached to the mating device with a thermal rod which hardens on contact. Once the screw has been mated, torque is used to realise the screw from the mating device.

Shu et al. (2011) echo the conclusions of Tinsley et al., they maintain that many of nature's solutions are so ingenious that engineers are now turning to biological solutions for inspiration when faced with engineering problems. Shu et al. pull together an extensive list of academic papers which demonstrate the deeper potential biomimicry holds for engineering. They argue that engineers should not only look to natural designs for potential solutions, but that they should apply principles, e.g. evolutionary algorithms, to create new designs which are inspired by nature's principles.

Traditional biomimicry is involved the emulation of biological solutions to analogous engineering problems, e.g. prosthetics. For the purpose of this paper, functional biomimicry will refer to situations where physical engineering design solutions have been directly motivated by nature, e.g. shark skin inspired wet suits (Rodgers, 2011). Mayer \& Sarikaya (2002) detail several examples where biological structures have been directly copied and applied to similar engineering problems, for example, the inner structure of a vulture's wing being applied to the structure of plane wings. Li et al. (1995) demonstrated a $15 \%$ increase in the sheer strength of several engineering materials through emulating the structure of bamboo fibre. Such examples can now be seen throughout engineering ranging from the examples detailed by Mayer and Sarikaya to building climate control systems based on termite mounds (Turner \& Soar, 2008).

\subsection{Research context \& Non-Functional Biomimicry}


Cleary, certain pattern formations are prevalent throughout nature and certainly patterns arise out of chaos. These patterns have formed complex living organisms which have been subjected to Darwinian evolution. As such, organisms have been subjected to a continuous struggle to reproduce. The result is organisms which have developed ingenious adaptations in order to address natural problems. Engineers can emulate these adaptations to solve analogous engineering issues, however, there is a growing consensus within the scientific community that, in order to fully utilise biomimicry, researchers must look beyond emulation and seek to understand and apply the principles behind biological design. Given that, if utilized correctly, biomimicry can provide clear benefits to functional design the hypothesis that biomimicry could be equally applied to aesthetic design seems inescapable. Consequently, the authors of this paper argue that biomimicry, if utilized properly, could provide similar benefits to aesthetic design. For the purpose of this paper, the application of biomimicry to the aesthetic aspects of a product will be defined as non-functional biomimicry.

\section{Experimental study}

An experiment was developed in order to focus on utilizing the potential to exploit the underlying geometrical structure of natural patterns in order to control aesthetic responses. An environment was developed using the virtual world platform Second Life (SL). Nino (2007) and Kirkpatrick (2006) consider SL to be a revolutionary method of communicating and displaying information over the internet. They maintain that SL facilitates a dimension of userinteraction that, until its development, was not available over the internet. Bates (2008) concurs, arguing that SL has the potential to redefine the how the internet is used.

The environment consists of a room which contains natural and unnatural patterns in the form of mounted pictures. Specifically, the room contains branching patterns, fractal patterns and spiral patterns. The room also contains pictures which appear similar in essence to the previously mentioned patterns, however, they are not authentic branching, fractal or spiral patterns. Six images of both types (natural and unnatural) were placed in a random order throughout the room and appeared, to the evaluators, to be roughly the size of a large house painting. The evaluators were free to explore the environment and, if desired, approach and interrogate the images in greater resolution through zooming into the images. Eighteen evaluators carried out the experiment.

The experiment was run under closed conditions, i.e. the only people present were the current evaluator and author who was conducting the experiment. Each evaluator was given a demonstration of, and time to familiarise themselves with, the controls in a different virtual environment. During the running of the experiments the author sat out of view of the evaluators and the evaluators were instructed to not communicate or interact with the author until the experiment was complete.

All of the experiments were videoed and captured using screen capture software. The footage was then replayed at a later date in order to determine the viewing times. The viewing times were calculated by overlapping the video data and screen captures, determining the position of 
the individual with the environment and evaluators' eye contact. Viewing was only recorded when the avatar was pointing directly at an individual image, i.e. that was the only whole image in the screen and the evaluators had clear eye contact with the screen.

\section{Selected images:}

\subsection{Development}

After carrying out a pilot test, several aspects of the environment and evaluation were altered. The design was based around three major changes aimed at removing the potential of user pathways to be determined by the platform design.

- Firstly, a single continuous shape was discarded in favour of a platform constructed of numerous shapes in order to make it impossible for the users to simply view all the images in series without being presented with a clear opportunity to view other images.

- Secondly, the overall size of the platform was reduced by over fifty percent. This ensured that, even at the furthest distance, all images were clearly viewable.

- Finally, two thirds of the images were set at a forty five degree angle to ensure that the two outside images on an island occupied an equally prominent position as the centre image. (Figure 4).

Another aspect of the experiment that was modified due to the pilot test results was the initial information which was provided to the user at the start of the evaluation. The lack of information during the pilot study resulted in the evaluators initially hypothesising the nature of the test and then behaving in accordance with their hypothesis.

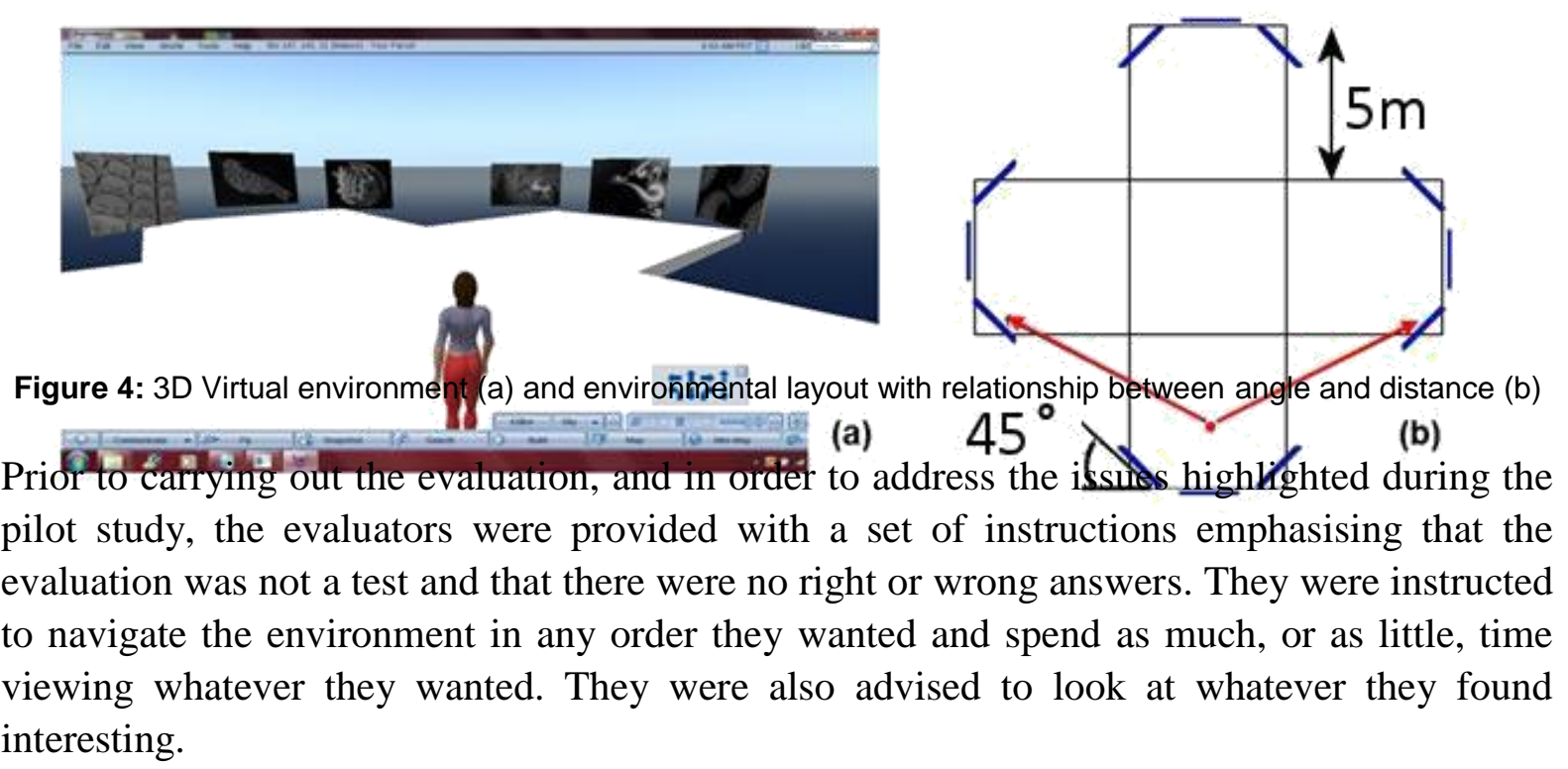

In order to reduce the potential of interface issues contaminating results, the evaluators were 
fully instructed on how to interact with the environment and advised that they could ask for assistance at any point throughout the evaluation. After the initial evaluation, the evaluators were then returned to the room and asked to rate each picture on a scale of 1-10 for interest.

The following hypotheses were formulated:

Primary Hypothesis: The underlying geometry of natural patterns will evoke an involuntary attention response from the majority of evaluators.

Secondary Hypothesis: The evaluators will demonstrate an increased interest towards underlying geometry of natural patterns.

The following null hypotheses were formulated in order to evaluate whether the produced data supports the initial hypotheses:

Null Hypothesis 1: There will be no relationship between natural patterns and attention response (viewing time).

Null Hypothesis 2: There will be no relationship between interest ratings and natural patterns. 


\section{Results}

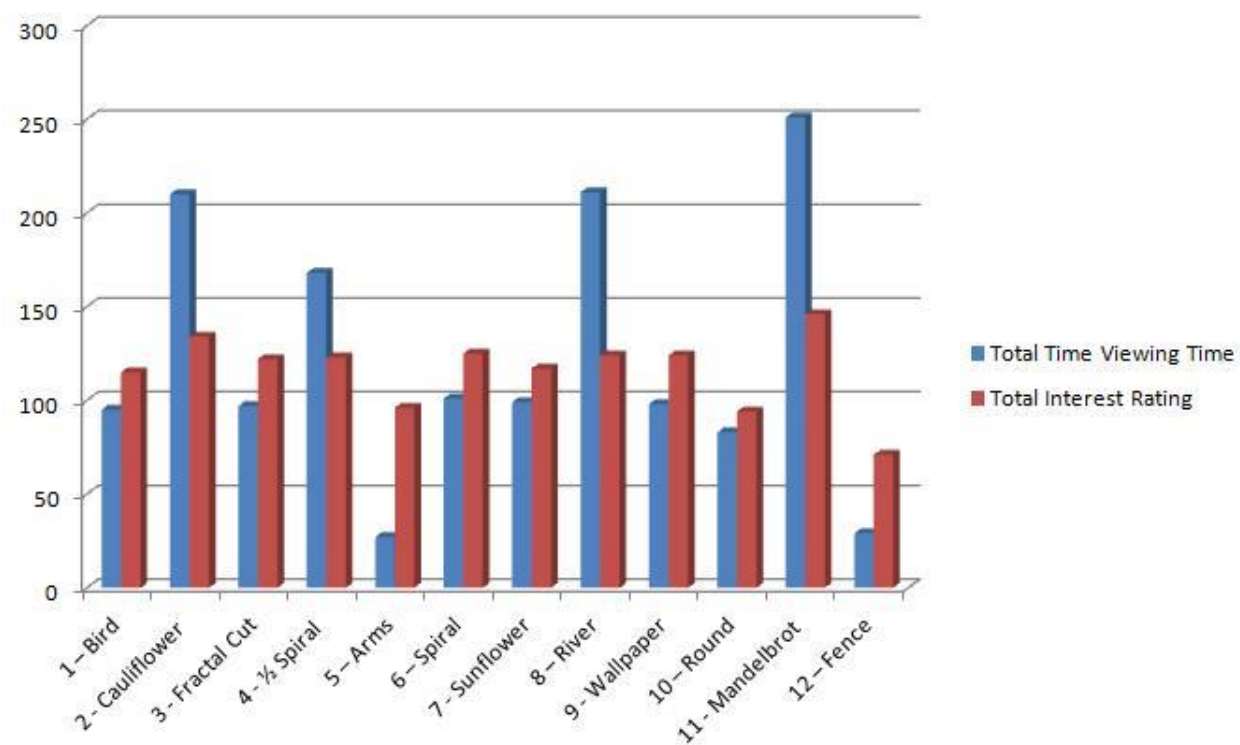

Figure 5 - Evaluator viewing times and interest ratings

A standard score (z-score) was calculated for each viewing time using the following formula:

$$
Z=\frac{X-\mu}{\sigma}
$$

$\mathrm{Z}=$ the standard score, $\mathrm{X}=$ viewing time per image, $\mu=$ the mean, $\sigma=$ the standard deviation

The z-score was cross referenced to the standard normal distribution table in order to calculate the probability that the viewing results were due to random chance, producing the following results.

Table 1 - Viewing time probabilities - Pattern types are coloured as follows: Red (Fractal); Blue (Branching); Green (Spiral).

\begin{tabular}{|l|c|c|c|}
\hline \multicolumn{1}{|c|}{ Image } & Viewing Time & $\underline{\text { Z-Score }}$ & Probability \\
\hline 1- Bird & $\mathbf{9 5}$ & $\mathbf{- 0 . 3 8 2 5}$ & $\mathbf{0 . 3 5 2}$ \\
\hline 2 - Cauliflower & $\mathbf{2 1 0}$ & $\mathbf{1 . 2 2 1 9 0 2}$ & $\mathbf{0 . 1 1 1 2}$ \\
\hline 3 - Fractal Cut & $\mathbf{9 7}$ & $\mathbf{- 0 . 3 5 4 6}$ & $\mathbf{0 . 3 5 9 4}$ \\
\hline 4 - 1/2 Spiral & $\mathbf{1 6 8}$ & $\mathbf{0 . 6 3 5 9 4 7}$ & $\mathbf{0 . 2 6 1 1}$ \\
\hline 5 - Arms & $\mathbf{2 7}$ & $\mathbf{- 1 . 3 3 1 1 9}$ & $\mathbf{0 . 0 9 1 8}$ \\
\hline 6- Spiral & $\mathbf{1 0 1}$ & $\mathbf{- 0 . 2 9 8 7 9}$ & $\mathbf{0 . 3 8 2 1}$ \\
\hline 7 - Sunflower & 99 & $\mathbf{- 0 . 3 2 6 6 9}$ & $\mathbf{0 . 3 7 4 5}$ \\
\hline 8- River & $\mathbf{2 1 1}$ & $\mathbf{1 . 2 3 5 8 5 3}$ & $\mathbf{0 . 1 0 7 5}$ \\
\hline 9- Wallpaper & $\mathbf{9 8}$ & $\mathbf{- 0 . 3 4 0 6 4}$ & $\mathbf{0 . 3 6 6 9}$ \\
\hline 10- Round & $\mathbf{8 3}$ & $\mathbf{- 0 . 5 4 9 9 1}$ & $\mathbf{0 . 2 9 1 2}$ \\
\hline 11 - Mandelbrot & $\mathbf{2 5 1}$ & $\mathbf{1 . 7 9 3 9 0 5}$ & $\mathbf{0 . 0 3 6 7}$ \\
\hline 12- Fence & $\mathbf{2 9}$ & $\mathbf{1 . 3 0 3 2 8}$ & $\mathbf{0 . 0 9 6 8}$ \\
\hline
\end{tabular}


In order to reach statistical significance probability must be $\mathrm{P}<=0.05$. A paired t-test was carried out on the total viewing time for both groups, producing the following results:

Table 2: T-test viewing time results

\begin{tabular}{|c|c|c|}
\hline Group & Group One & Group Two \\
\hline Mean & 71.50 & 173.33 \\
\hline SD & 34.13 & 62.58 \\
\hline SEM & 13.93 & 25.55 \\
\hline N & 6 & 6 \\
\hline
\end{tabular}

The two-tailed $\mathrm{P}$ value equals 0.0057 . By conventional criteria, this difference is considered to be very statistically significant. Consequently, the initial null hypothesis 'There is no relationship between natural patterns and attention response' cannot be rejected.

Applying the same process to the interest ratings, i.e. the interest ratings are due to random preference with no underlying factors controlling preference produces the following results.

Table 3 - Interest rating probabilities - Pattern types are coloured as follows: Red (Fractal); Blue (Branching); Green (Spiral).

\begin{tabular}{|c|c|c|c|}
\hline Image & Interest Rating & Z-Score & Probability \\
\hline $1-$ Bird & 115 & -0.04568 & 0.2546 \\
\hline 2-Cauliflower & 134 & 0.90121 & 0.1841 \\
\hline 3 - Fractal Cut & 122 & 0.303172 & 0.3821 \\
\hline 4 - 1/2 Spiral & 123 & 0.353008 & 0.3632 \\
\hline $5-$ Arms & 96 & -0.9925 & 0.1611 \\
\hline 6-Spiral & 125 & 0.452681 & 0.3264 \\
\hline 7 - Sunflower & 117 & 0.053989 & 0.4801 \\
\hline $8-$ River & 124 & 0.402845 & 0.3446 \\
\hline 9- Wallpaper & 124 & 0.402845 & 0.3446 \\
\hline $10-$ Round & 94 & -1.09225 & 0.1375 \\
\hline 11 - Mandelbrot & 146 & 1.499248 & 0.0668 \\
\hline $12-$ Fence & 71 & -2.23849 & 0.0125 \\
\hline
\end{tabular}

A paired t-test was carried out on the total viewing time for both groups, producing the following results:

Table 4: T-test interest rating results

\begin{tabular}{|c|c|c|}
\hline Group & Group One & Group Two \\
\hline Mean & 103.67 & 128.17 \\
\hline SD & 20.48 & 10.30 \\
\hline SEM & 8.36 & 4.21 \\
\hline N & 6 & 6 \\
\hline
\end{tabular}


The two-tailed P value equals 0.0924. By conventional criteria, this difference is considered to be not quite statistically significant. Consequently, the second null hypothesis "There is no relationship between interest (ratings) and natural patterns.' can be rejected.

\subsection{User Pathways}

The evaluators' pathways were recorded by noting the order, using the picture numbers in which the evaluators viewed the images. The following results were produced:

The above data was then superimposed onto a map of the environment in order to determine if there was any correlation between the user pathways and the images. Two of the user paths (user $16 \&$ user 17) were not superimposed onto the map due to the fact that they simply viewed all the images in order, therefore, removing the possibility for the images to influence the paths they used to circumvent the environment.

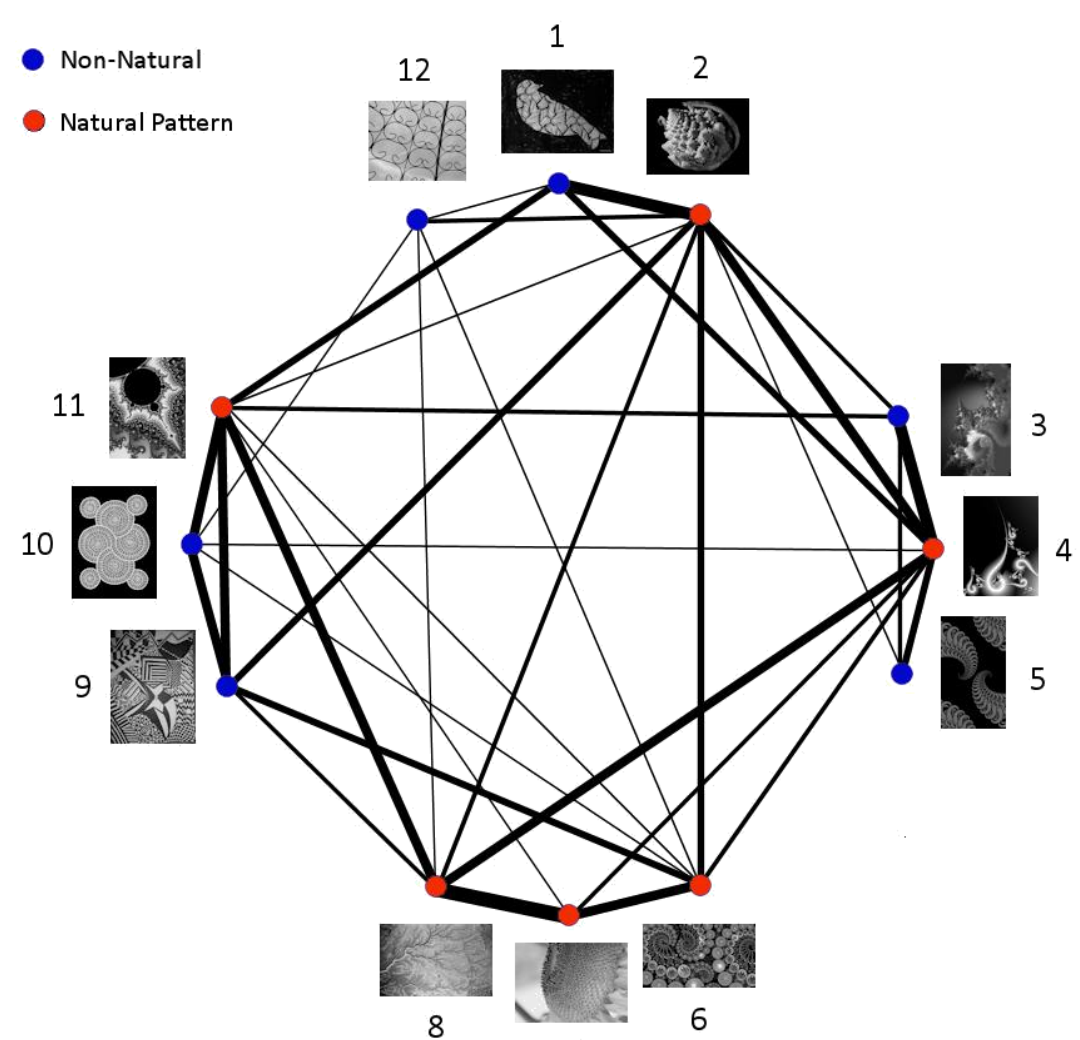

Figure 6: Evaluators Navigation Pathways

The user pathways (Figure 6) reveal the following regarding the manner in which the evaluators navigated the environment:

- The evaluators had the tendency to view images in close proximity. There is not a single case of any image that is not linked to an image directly next to it (i.e. 6 and 7, 4 and 5 , etc) by a user pathway. The user pathways, however, suggest that the natural patterns have the potential to override such behaviour. For example, after viewing image 12, all but one of the evaluators skipped viewing the adjacent image (image 1) in favour of 
viewing the natural pattern contained in image 2 . The same behaviour can also be seen between images 9, 10, and 11. Furthermore, images 3, 4, and 5 demonstrate that above behaviour is not due to the layout of the environment, as all but one of the evaluators go directly from image 3 to 4 , to the natural pattern.

- Another interesting set of relationships is of that between 8 and 11 and 4 and 8 . In these cases, it seems reasonable to argue that the evaluators are navigating to images based on their influence. One could argue, however, that the images are enticing the evaluators due to the fact that they occupy a more prominent position. If that were the case, one would expect to see the same relationships between images 5 and 8, 2 and 5, and 7 and 10, however no such relationships exist. Furthermore, image 11 and 8 is one of the only examples where we see a reverse relationship, i.e. evaluators moving from 8 to 11 which given the layout of the images is a noteworthy result.

- Images 2 and 6, 3 and 11, 12 and 8, 5 and 9 also support the hypothesis that images are influencing the user pathways. In these cases, the images are directly opposite each other, with 3 out of the 4 relationships containing natural patterns ( 2 and 6, 3 and 11, 12 and 8). There are clear user pathways between the 3 relationships with the natural patterns, however, none of the users moved between images 5 and 9.

\section{Results Discussion}

\subsection{Primary Hypothesis}

The underlying geometry of natural patterns will evoke an involuntary attention response from the majority of participants.

The initial results support the primary hypothesis. Of the twelve images, the six which contained underlying natural geometry were the six most viewed images with the evaluators viewing the natural patterns for, when grouped and averaged, 241\% longer than the non-natural patterns. The viewing results produced probabilities to a sufficient degree of statistical significance to reject the null hypothesis 'There is no relationship between natural patterns and attention response (viewing time).' The initial hypothesis, however, was not just that patterns would influence responses but that they would evoke involuntary responses.

To this end, perhaps the most interesting results were produced by the river image which produced an interesting rating probability of 0.35 and a viewing probability or 0.1 , i.e. the evaluators did not find the image to be overly interesting, however, the image produced an intriguingly large attention response which narrowly missed out on statistical significance. Furthermore, bland images, e.g. the gate, which had no underlying geometrical patterns, were shown, to statistical significance, to have an extremely limited ability to evoke attention responses.

The gender of evaluators produced similar results with both the male and female evaluators sharing identical viewing preferences. The user pathways also supported the hypothesis, with the pathways producing relationships which suggest that the natural patterns were influencing the manner in which the user navigated the environment. 


\subsection{Secondary Hypothesis}

The evaluators demonstrated an increased interest towards underlying geometry of natural patterns. The interest rating probabilities of the natural patterns, however, did not achieve statistical significance and, therefore, achieve a probability level sufficient enough to reject the null hypothesis 'There is no relationship between interest (ratings) and natural patterns' As above, the natural patterns occupied the top ratings for interest, with six out of the top seven interest ratings being natural patterns.

The results, however, demonstrated a significant correlation to a probability of $\mathrm{P}=0.01$, between interest ratings and viewing times, suggesting that not only did the natural patterns evoke attention responses but that the interest responses also had a significant impact on the viewing times. Furthermore, the total number of times that the images were viewed, another possible measure of interest, was also heavily influenced by the natural patterns with the six natural patterns occupying the top six places.

The female interest ratings produced interesting results in relation to viewing times. Several of the non-natural patterns produced interest ratings which rivalled the natural patterns, however, the viewing results did not, i.e. although the individuals claimed equal interest in the natural/non-natural patterns, they viewed the non-natural patterns for significantly longer. This suggests the natural patterns evoked involuntary attention responses from the viewers.

\section{Conclusion}

Natural reoccurring patterns arise from chaos and are prevalent throughout nature. The formation of these patterns is controlled by, or produces, underlying geometrical structures. These patterns have influenced human evolution where the recognition of such patterns provides a survival advantage through impacting the manner in which hominids interacted with the main evolutionary factors i.e. prey acquisition, predator avoidance, necessities, and reproduction. As such, these patterns now have the ability to affect humans on numerous levels, e.g. vegetation and relaxation. Consequently, these patterns may provide and basis for, and explanation of, the nature of aesthetics.

An experiment was conducted to investigate the impact of natural patterns on attention responses. Evaluators were asked to navigate through a virtual environment that contained a mixture of natural patterns (e.g. branching, spiral, and fractal) and pseudo patterns, i.e. patterns that did not contain such underlying geometrical structures. The results showed that natural patterns have the ability to invoke attention responses to a statistically significant level and a significant correlation, to a probability of $\mathrm{P}=0.01$, was demonstrated between interest ratings and viewing times. Furthermore, a high attention response was achieved in the case where the individuals did not consider the natural pattern to be particularly interesting. The results imply that natural patterns have the ability to influence the manner in which the evaluators navigated the environment with the pathways producing relationships which suggested that the natural patterns were influencing the manner in which the user navigated the environment.

More broadly, results of this research support the hypothesis that individuals are evolved to 
respond to natural patterns and that the underlying geometrical structure of patterns possess the ability to evoke attention and interest responses.

\section{Acknowledgements}

The authors would like to thank Kim Williams for proofreading the document.

\section{References}

Ball, P., \& Borley, N. R. (1999). The self-made tapestry: pattern formation in nature (Vol. 
198). Oxford: Oxford University Press.

Ball, P. (2008). Water: water-an enduring mystery. Nature, 452(7185), 291-292.

Ball, P. (2011). Shapes: nature's patterns: a tapestry in three parts (Vol. 1). Oxford University Press.

Ball, P. (2008). Water: water — an enduring mystery. Nature, 452(7185), 291-292.

Bates, M. E. 2008, “The three-dimensional internet”, EContent, 31(7), 52-52.

Benyus, J., (2002), Biomimicry: Innovation Inspired by Nature, William Morrow, New York

Bottino, A., Laurentini, A., Campilho, A. \& Kamel, M. (2010). The Analysis of Facial Beauty: An Emerging Area of Research in Pattern Analysis

Camazine, S., J. Deneubourg, H. R. Franks, J. Sneyd, G. Theraula, and E. Bonabeau, (2001) Self-Organization in Biological Systems. Princeton University Press, Princeton, NJ.

Chamberlain, A. T. (2000). On the evolution of human aesthetic preferences. Assemblage: University of Sheffield Graduate Student Journal of Archaeology, 5.

Cook TA. 1979. The Curves of Life. New York: Dover Publ.

Dafni A, L. Efraim, S. Beckman, C. Eichberger, (2006) Ritual plants of Muslim graveyards in northern Israel J. Ethnobiol. Ethnomed., 2 (2006), pp. 38-46

Dafni, A., (2007), The supernatural characters and powers of sacred trees in the Holy Land,cInstitute of Evolution, the University of Haifa, Haifa 31905, Israel

Dawkins, R., (1986) The blind watchmaker: Why the evidence of evolution reveals a universe without design, Norton \& Company

Dawkins, R. (1997). Climbing mount improbable. WW Norton \& Company.

Dawkins, R., (2006) The Selfish Gene, Oxford University Press

Devaney R.L. (1999). The Mandelbrot set and the Farey tree and Fibonacci sequences. American Mathematical Monthly 1999;10(6):286-302.

Dunlap, R.A. (1988). The Golden Ratio and Fibonacci Numbers, World Scientific, Singaproe. Dutton, D., (2009), "The Art Instinct." Oxford: Oxford UP, forthcoming.

Enquist, M., \& Arak, A. (1994). Symmetry, beauty and evolution. Nature, 372(6502), 169172.

Falk, J. H. \& Balling, J. D. (2010). Evolutionary Influence on Human Landscape Preference. Environment and Behavior, 42, 479-493. 
Han, K. T., (2007), Responses to six major terrestrial biomes in terms of scenic beauty, preference, and restorativeness. Environment \& Behavior

Hazen R. M. (2009), The emergence of patterning in life's origin and evolution. Int J Dev Biol. 2009;53:683-692. doi: 10.1387/ijdb.092936rh.

Hazen, R. M., Griffin, P., Carothers, J. and Szostak, J. (2007). Functional information and the emergence of biocomplexity. Proc Natl Acad Sci USA 104: 8574-8581.

Hernandez, S., \& Brebbia, C. A. (2012). Design and nature VI: comparing design in nature with science and engineering (Vol. 4). WIT press.

Holldobler, B., and E. O. Wilson, (1990), The Ants. Berlin: Springer-Verlag

Jones, B. C., Little, A. C., Penton-Voak, I. S., Tiddeman, B. P., Burt, D. M., \& Perrett, D. I. (2001). Facial symmetry and judgements of apparent health: Support for a 'good genes' explanation of the attractiveness-symmetry relationship. Evolution and Human Behavior, 22, 417-429.

Joye, Y., (2007) Architectural lessons from environmental psychology: the case of biophilicarchitecture.

Joye, Y., Poels, K., Willems, K. \& Saad, G. (2011). "Evolutionary Store Atmospherics" Designing with Evolution in Mind

Judelman, G., (2004), "Aesthetics and inspiration for visualization design: bridging the gap between art and science," Information Visualisation, 2004. IV 2004. Proceedings. Eighth International Conference on , vol., no., pp. 245- 250, 14-16 July.

Kaya, N. and Crosby, M. (2006), Color associations with different building types: An experimental study on American college students. Color Res. Appl., 31: 67-71. doi: $10.1002 / \mathrm{col} .20174$

Kellert, S. R., \& Wilson, E. O. (1995). The biophilia hypothesis. Island Press.

Klein, L. (2009). A phenomenological interpretation of Biomimicry and its potential value for sustainable design (Doctoral dissertation, Kansas State University).

Kirkpatrick, D., 2006, "No, Second Life is not overhyped", Fortune Magazine [online] Available from http://money.cnn.com/2006/11/09/technology/fastforward_secondlife.fortune/index.htm [Accessed January 19th 2010]

Leder, H. and Carbon, C.-C. (2005), Dimensions in appreciation of car interior design. Appl. Cognit. Psychol., 19: 603-618. doi: 10.1002/acp.1088

Li, S. H., Zeng, Q. Y., Xiao, Y. L., Fu, S. Y., \& Zhou, B. L. (1995). Biomimicry of bamboo bast fiber with engineering composite materials. Materials Science and Engineering: C, 3(2), 
125-130.

Liu, R., Liaw, S., \& Maini, P. (2006). Two-stage Turing model for generating pigment patterns on the leopard and the jaguar. Physical Review E, 74(1), 11914.

Livio, M. (2008). The golden ratio: The story of phi, the world's most astonishing number. Broadway Books.

Lynn, R., Vanhanen, T., (2006) IQ and Global Inequality, a sequal to IQ and the Wealth of Nations.

Mandelbrot, B.B., (1983), The fractal geometry of nature, W. H. Freeman and co., New-York

Mandelbrot, B. B. (1998), Is nature fractal? Science ;279:783-4.

Mayer, G., \& Sarikaya, M. (2002). Rigid biological composite materials: structural examples for biomimetic design. Experimental Mechanics, 42(4), 395-403.

Meinhardt, H. (1995), "The Algorithmic Beauty of Sea Shells", Springer

Millonas MM., Rauch EM, (2004), The role of trans-membrane signal transduction in turingtype cellular pattern formation. J. Theor. Biol. 2004;226:401-407.

Oberascher L. and Gallmetzer M., (2003), "Colour and emotion.” Proceedings of AIC 2003 Bangkok: Color Communication Management, 370-374.

Orians, G. H., (1986). An ecological evolutionary approach to landscape aesthetics. In E. C.

Orians G. H., Heerwagen J. H. Barkow,J. H., Cosmides,L., ToobyJ. (1992). Evolved responses to landscapes. The adapted mind: Evolutionary psychology and the generation of culture. (pp. 555-579). New York: Oxford University Press.

Ouyang, Q. and Swinney, H.L. (1991). Transition from a uniform state to hexagonal and striped Turing patterns. Nature 352: 610-612.

Passino, K., M., (2004) Biomimicry for Optimization, Control, and Automation. New York, Springer-Verlag

Paul S. T., Morris, Olausson, M. S., Reed., (2011), Effects of Apophenia on Multiple-Choice Exam Performance, Robert Morris University

Peitgen, H. 0., Richter. P. H., (1986), The Beauty of Fractals. New York: Springer

Pickett, S. T., \& White, P. S. (Eds.). (2013). The ecology of natural disturbance and patch dynamics. Elsevier.

Prusinkiewicz, P., \& Lindenmayer, A. (2012). The algorithmic beauty of plants. Springer Science \& Business Media.

Ramachandran, V. S., \& Hirstein, W. (1999). The science of art: A neurological theory of 
aesthetic experience. Journal of Consciousness Studies

Richards, R. (2001). A new aesthetic for environmental awareness: Chaos theory, the beauty of nature, and our broader humanistic view. Journal of Humanistic Psychology, 41(2), 59-95.

Rietkerk, M., \& Van de Koppel, J. (2008). Regular pattern formation in real ecosystems. Trends in Ecology \& Evolution, 23(3), 169-175.

Rhodes, G., Proffitt, F., Grady, J. M., Alexsumich, (1998), Facial symmetry and the perception of beauty, Psychomet. Bull. Rev., 5 (1998), pp. 659-669

Rhodes, G., Zebrowitz, L. A., Clark, A., Kalick, S. M., Hightower, A., \& McKay, R. (2001). Do facial averageness and symmetry signal health? Evolution and Human Behavior, 22, 31-46.

Rhodes, G., Simmons, L. W. \& Peters, M. (2005). Attractiveness and sexual behaviour: Does attractiveness enhance mating success? 26, 186-201.

Rhodes, G., (2006), The evolutionary psychology of facial beauty, Annual Review of Psychology 57: 199-226.

Rogers K. S., (2011) The Handbook of Knowledge-Based Coaching, Wiley

Sharma, V., Joshi, B.D., (2010), Role of Sacred Plants in Religion and Health-care system of local people of Almora district of Uttarakhand State (India), Academic Arena 2010;2(6)

Shu, L. H., Ueda, K., Chiu, I., \& Cheong, H. (2011). Biologically inspired design. CIRP Annals-Manufacturing Technology, 60(2), 673-693. Steadman, P. (2008). The Evolution of Designs: Biological analogy in architecture and the applied arts. Routledge.

Sutcliffe, A. \& Johnson, C. 2001. Heuristic Evaluation of Website Attractiveness and Usability

Swaddle, J.P. \& Cuthill, I.C. (1995). Asymmetry and human facial attractiveness: Symmetry may not always be beautiful. Proceedings: Biological Sciences, 261(1360), 111-116.

Teuscher., C, (2004)., Life and Legacy of a Great Thinker, Springer

Tinsley, A., Midha, P. A., Nagel, R. L., McAdams, D. A., Stone, R. B., \& Shu, L. H. (2007, January). Exploring the use of functional models as a foundation for biomimetic conceptual design. In ASME 2007 International Design Engineering Technical Conferences and Computers and Information in Engineering Conference (pp. 79-92). American Society of Mechanical Engineers.

Thompson, D. W. (1915). On growth and form. Cambridge: University Press.

Ulrich R. S., (1981). Natural versus urban scenes: some psychophysiological effects. Environment and Behavior, Evironment and Behavior, 13, 523-556.

Ulrich, R.S., Simons, R.F., Losito, B.D., Fiorito, E., Miles, M.A., Zelson, M., (1991). Stress recovery during exposure to natural and urban environments. J. Environ. Psychol. 11, 201230. 
Wannarumon S., Bohez, E.L.J. (2006). A new aesthetic evolutionary approach for jewelry design. Computer-Aided Design \& Applications, 3(1-4): 385-394

Williams, G. C. (1966). Natural selection, the costs of reproduction, and a refinemento $\mathrm{f}$ Lack'sp rinciple.- Am. Nat. 100: 687-690.

Wilson, E. O. (1984). Biophilia. Harvard University Press.

Zaidel, D.W.; Hessamian, M. (2010), Asymmetry and Symmetry in the Beauty of Human Faces. Symmetry 2010, 2, 136-149. 